\title{
Note on references and citations
}

- The works referred to in the footnotes are identified by full bibliographic references in their first citation, and thereafter in abbreviated form;

- Absent any other reference, the statutory texts cited correspond to the versions available on the Internet sites mentioned in $\S 3, f)$;

- Where no other source is indicated, the court rulings cited in the text were accessed from the databases indicated in $\S 3, g$ );

- All translations of texts originally published in languages other than English are, unless otherwise indicated, the author's responsibility. 